DEMOGRAPHIC RESEARCH

VOLUME 28, ARTICLE 39, PAGES 1145-1166

PUBLISHED 30 MAY 2013

http://www.demographic-research.org/Volumes/Vol28/39/

DOI: 10.4054/DemRes.2013.28.39

Reflection

\title{
Very long range global population scenarios to 2300 and the implications of sustained low fertility
}

Stuart Basten

Wolfgang Lutz

Sergei Scherbov

(C) 2013 Stuart Basten, Wolfgang Lutz \& Sergei Scherbov.

This open-access work is published under the terms of the Creative Commons Attribution NonCommercial License 2.0 Germany, which permits use, reproduction \& distribution in any medium for non-commercial purposes, provided the original author(s) and source are given credit.

See http:// creativecommons.org/licenses/by-nc/2.0/de/ 


\section{Table of Contents}

1 Introduction 1146

$2 \quad$ Could global fertility levels fall to well below population 1147 replacement level?

3 Method 1151

$4 \quad$ Results 1152

$5 \quad$ Conclusions 1154

6 Acknowledgement 1156

$\begin{array}{ll}\text { References } & 1157\end{array}$

Appendix 1: Detailed results of alternative global population 1162 projections to 2300

Appendix 2: Input data, code and method of calculation 1165 


\title{
Very long range global population scenarios to 2300 and the implications of sustained low fertility
}

\author{
Stuart Basten ${ }^{1}$ \\ Wolfgang Lutz ${ }^{2}$ \\ Sergei Scherbov ${ }^{3}$
}

\begin{abstract}
BACKGROUND

Depending on whether the global level of fertility is assumed to converge to the current European TFR ( 1.5) or that of Southeast Asia or Central America ( 2.5), global population will either decline to 2.3-2.9 billion by 2200 or increase to 33-37 billion, if mortality continues to decline. Furthermore, sizeable human populations exist where the voluntarily chosen ideal family size is heavily concentrated around one child per woman with TFRs as low as $0.6-0.8$. However, the UN population projections to 2300 use a much narrower band of possible future TFRs.
\end{abstract}

\section{OBJECTIVE}

If the two-child norm is not necessarily the end-point of transition, what would be the consequences of the currently reported low fertility rates being sustained and becoming widespread?

\section{METHODS}

We present new projections for 13 IPCC world regions with scenarios calculated on the basis of regular cohort-component projections by age and sex in single-year time steps up to 2300, each based upon a much broader set of fertility assumptions than currently employed. We create three mortality scenarios based upon maximum life expectancies of 90,100 , and 110 , as well as a series of 'special' scenarios.

1 Department of Social Policy and Intervention, University of Oxford, United Kingdom. E-mail: stuart.basten@spi.ox.ac.uk.

${ }^{2}$ Wittgenstein Centre for Demography and Global Human Capital (IIASA, VID/ÖAW, WU), International Institute for Applied Systems Analysis, Austria.

${ }^{3}$ Wittgenstein Centre for Demography and Global Human Capital (IIASA, VID/ÖAW, WU), International Institute for Applied Systems Analysis, Austria. 


\section{RESULTS}

Even under conditions of further substantial increases in life expectancy, world population size would decline significantly if the world in the longer run followed the current examples of Europe and East Asia.

\section{CONCLUSIONS}

In contrast to Malthusian disaster scenarios, our exercise illustrates the distinct possibility of significant population shrinking associated with increasing life expectancy and human well-being.

\section{Introduction}

I don't want a second child. One is enough, and I hope it is a girl. It is very nice to be the only child; you don't need to share or grab things from others. You can have all your parents' attention. My parents have brothers and sisters, but when my grandparents died they quarrelled over the legacy. That was horrible and hurtful. Being the only child, you won't have those problems.

25-year-old Shanghainese expectant mother (Branigan 2009)

The future number of humans on our planet will crucially depend on the future level of human reproduction in different parts of the world, which is uncertain. Depending on whether the global level of fertility is assumed to converge to the current European TFR $(\sim 1.5)$ or those of parts of Southeast Asia or Central America ( 2.5), global population will either decline to 2.3-2.9 billion by 2200 or increase to 33-37 billion, if mortality continues to decline. The process of demographic transition has resulted in universal fertility declines (with the global TFR declining from 4.9 in the 1960s to 2.6 currently), with the decline failing to halt at a replacement level of around 2.1 in many countries, as was anticipated by earlier international projections. The key question is how low fertility can fall in modern societies where the key determinant may lie in social norms about ideal family size.

"People will always have children" is a quote attributed to both Winston Churchill and Konrad Adenauer (Abrahamson, Boje, and Greve 2005). The latter supposedly said it in reaction to an expert pointing out that the German pay-as-you-go pension system was rather vulnerable to the possibility of declining birth rates. This was in the early 
1960s, when the Baby Boom was at its height and the TFR was well above the replacement level of 2.1. Soon thereafter, fertility rates in Germany entered a steep and lasting decline - currently hovering around 1.4, or two thirds of replacement level putting the pension system under severe stress. Was Adenauer wrong?

While most existing world population projections agree that we are likely to see the end of world population growth (with a peak population of between eight and ten billion) during the second half of this century due to the on-going process of demographic transition from high birth and death rates to low ones, little has been said about the longer term future. The United Nations Population Division published projections for all countries until 2300 based on alternative fertility assumptions (UNPD 2004). However, the range of possible future fertility levels was extremely narrow, with the lowest level considered assuming a long term TFR of 1.85 and the highest scenario assuming 2.35. For the medium scenario exact replacement fertility is assumed which, by definition, results in long term constancy of population size in every country. Given that the long term 'floor' figure of 1.85 is significantly higher than the fertility Europe has experienced over the past 30 years and the recent ultra-low fertility experiences of East Asia, we sought to widen the scope of the very long-term projections by presenting an alternative set of convergence futures which range from the extremes of a longer term stalled fertility decline in Sub-Saharan Africa to the scenario of the whole world moving toward some of the lowest national TFRs currently found in the world.

\section{Could global fertility levels fall to well below population replacement level?}

Fertility levels well below replacement rate have been in evidence in many world regions for a number of decades. In Europe, traditionally thought of as being a vanguard in the transition to sub-replacement fertility, a large number of countries experienced 'lowest-low' fertility in the 1990s and early 2000s - defined as a period TFR of below 1.3 (Kohler, Billari, and Ortega 2002). While most countries affected have seen their TFRs rise above this figure - not least through the 'tempo' effect of postponed childbearing on period measurement - the experience of low fertility made a profound impact upon both the policy and academic demographic discourse (Sobotka 2004; Frejka et al. 2008; Frejka and Sobotka 2008; Goldstein, Sobotka, and Jasilioniene 2009). Indeed, it is out of this new paradigm that a fundamental rethinking of some of the core precepts of the classical Demographic Transition model have developed, including the questioning of the two-child family model (Frejka 2008a). In 2002, therefore, when the UN broke from previous assumptions of convergence at around 
replacement and switched to 1.85 (UNPD 2003) - almost certainly in recognition of the new demographic regime in Europe - this was greeted as 'breaking the demographic sound barrier' (Wattenberg 2005) in terms of the global implications of belowreplacement fertility.

However, how can we interrogate possible future trajectories of fertility? There is little doubt that from an evolutionary perspective our sex drive has been the main mechanism assuring the reproduction of the human species and that modern contraception has radically changed this pattern (e.g. Frejka 2008b). In this context, individual desires, ideals, and social norms are paramount for the decision to have a child. Indeed, fertility ideals and intentions have been described as powerful predictors of future fertility behaviour (e.g. Morgan and Rackin 2010).

One of the strongest social norms regarding childbearing in Europe is that of the normative power of the two-child ideal. In the Eurobarometer survey of EU countries, the percentage of people reporting two children as an ideal family size over the decade 2001-2011 rose from 52\% to $57 \%$ for the general, or societal, ideal and from $49 \%$ to $52 \%$ for the personal ideal. By contrast, percentage of respondents aged 15-39 who state an ideal family size of one is just $10 \%$, with $4 \%$ stating a desire to remain childless (Testa 2012).

This evidence is especially important in terms of how long-term projections are explicitly, and implicitly, designed. While the latest round of the UN's global population projections to 2100 are based upon a probabilistic model of fertility change, the end point of the projection model fluctuates around an asymptotic mean at replacement rate (UNPD 2011a). The implicit assumption behind this, therefore, is that the 'end point' of fertility transition is at, or around, replacement rate. Empirically, this would appear to be based upon the experiences of Northern- and Western-Europe and North America, which have seen relatively stable cohort and tempo-adjusted fertility rates of around replacement for some years. Despite the fact that the recent economic crisis has stalled fertility increases in many parts of Europe, the strength of the twochild norm as reflected in the Eurobarometer data supports the idea of an end point of fertility transition at, or around, two children (Sobotka, Skirbekk, and Philipov 2012; Testa 2012).

But what of the ultra-low fertility rates in evidence in East Asia? Following extremely rapid fertility decline, TFRs in Hong Kong SAR, Japan, Singapore, South Korea, and Taiwan have reached a global nadir of below 1.3 (Straughan, Chan, and Jones 2008; Boling 2008). Furthermore, local statistical offices in the region are pessimistic about an upturn in the short- and medium- term (e.g. HKCSD 2012, Basten 2013).

Crucially, however, a growing body of evidence exists to suggest that fertility ideals and intentions have also fallen to levels significantly below replacement level. In 
Taiwan in 2003, the mean ideal number of children among Taiwanese women was reported to be 1.8 while in Hong Kong a 2011 survey of young people found the ideal number of children to be as low as 1.5 (Basten, Coleman, and Gu 2012). These are levels which are not currently observed in contemporary Europe. Furthermore, under the conditions of the so-called 'Low Fertility Trap' hypothesis, the normalisation of small family sizes as reflected through lower fertility ideals and intentions could mean that fertility rates become harder to raise in the future through family policy or other means (Lutz, Skirbekk, and Testa 2006).

There is a massive natural experiment unfolding in the cities of China which demonstrates the existence of sizable human populations who voluntarily elect not to conform to a two-child norm. After two decades of a strict one-child policy, many young couples are now allowed, but frequently choose not, to have more than one child.

According to the 2010 Chinese Census, the resident population of Shanghai Municipality is 23.02 million (NBS 2011) - roughly the same as Australia or Sweden, Finland, Norway, Estonia, and Latvia combined. The TFR of the city's registered population - which accounts for $72.6 \%$ of the total population - has steadily declined from 1.23 in 1979 to below 1.0 by 1994 and reaching a nadir of 0.64 in 2003 with a recent slight upturn to 0.88 by 2008 (SMPFPC 2009a, SMPFPC 2009b). By 2000, other large Chinese urban centers saw a similar decline in fertility rates - such as Beijing (0.69) (Hou and Ma 2008) and Tianjin (0.91) (Gu 2009).

These ultra-low fertility rates go together with low mean childbearing intentions among Shanghai's registered population, which, according to surveys, have fallen from 2.04 in 1983 to just 1.07 in 2008 (SMPFPC 2009c, SMPFPC 2008), despite the fact that most couples would be free to have two children under the official policy as they are both only children. Such ultra-low fertility intentions can also be seen in other parts of China such as Beijing (1.23) (Hou and Ma 2008; Hou, Ma, and Huang 2008), Nanjing (1.21) (Wen et al. 2005) and elsewhere (e.g. Basten, Coleman and Gu 2012, Zheng et al. 2009, Wen and Zong 2006).

As with a wide variety of topics found in social surveys, respondents may frame their answers based upon not just personal views but within the nexus of social norms and what might be termed 'politically correct' attitudes. While surveys from a wide variety of settings and contexts have found a general correlation between fertility intentions and outcomes (e.g. Morgan and Rackin 2010), it is possible to argue that the 'politically correct' motivation of respondents in China may be particularly strong especially given that the SMPFPC Survey was carried out by a Governmental organization. Hermalin and Liu (1990) compared face-to-face and anonymous methods of data collection of fertility preferences in the mid-1980s and found that the anonymous returns were generally higher, by up to 0.5 children. As such, even with this 
degree of uncertainty and building in such inflation, the levels reported for Shanghai are still well below replacement level.

Importantly, however, we can also identify the mean desired family size among the migrant population of Shanghai. This 'floating population' of workers hail from predominantly rural areas which are generally characterised by weaker family planning restrictions (such as the so-called 1.5-child policy where couples are allowed a second child if their first is a girl) and higher desired and realised fertility. However, even among this 'floating' population, the mean desired family size is just 1.30 among men and 1.36 among women (SMPFPC 2008).

While the Chinese family planning restrictions may be considered a unique episode in human history, it teaches us an important lesson that is relevant for the future of human reproduction: personal family size ideals can be greatly influenced by changing norms and social interaction and there can be situations for large populations in which the ideal - now voluntarily chosen and freely expressed - is heavily centred around just one (surviving) child.

Indeed, looking beyond East Asia we can see further fragmented pieces of evidence of ideal family sizes dropping below the European two-child norm. In India, for example, the 2005-06 National Family Health Survey found that 17.0\% of women stated a preference for just one child. Among urban women this rose to $24.5 \%$, while fully $30 \%$ of the richest and most educated women stated a preference for just one child (Basten and Kumara 2011).

In the World Fertility Survey and Demographic and Health Surveys, we can see that rapid declines in TFR have been accompanied by often dramatic falls in the ideal number of children desired. So much so that sub-replacement fertility ideals are already in evidence among younger cohorts in Vietnam, Bolivia, Peru, Colombia, El Salvador, Nepal (ICF International 2012), and Thailand (UNFPA 2011).

In conclusion, therefore, we suggest that the current paradigm of thinking around a two-child norm as the natural end point to fertility transition could, indeed, be challenged. This is especially so for countries whose recent economic, social, and educational development appears to more closely mirror the East Asian experience not least in speed of change - rather than the European experience. To put it another way, many East Asian economies have reached extremely high levels of industrial capacity with some of the highest educational attainment rates in the world - all of which have been achieved with remarkable speed. As countries in Southeast Asia and South America industrialise and post high levels of economic growth and rapid fertility decline, could their fertility transition end point not be closer to South Korea than to Sweden?

Of course, these questions are entirely hypothetical - but we suggest that there is enough evidence to at least justify the exercise of examining possible very-long range 
global population futures beyond the rather narrow ranges of the UN's projections, i.e. 'below 1.85'. In doing so, we can understand the long-term global consequences of different trajectories of fertility which could provide a counterpoint to much of the current discourse concerning the population 'explosion'.

\section{Method}

The calculations have all been carried out at the level of 13 world regions which follow the classification as used in the Intergovernmental Panel on Climate Change (IPCC) Special Report on Emissions Scenarios (Nakicenovic and Swart 2000). The scenarios have been calculated on the basis of regular cohort-component projections by age (single years of age) and sex in single-year time steps up to 2300. While some agencies have produced probabilistic projections to 2100 , we suggest that simply too little is known about living conditions and future technologies that would justify any probabilistic assessment beyond the end of the current century. As such, in order to maintain consistency in our methods we have utilised a deterministic cohort-component method for the purposes of this sensitivity analysis.

Until 2010 we used estimated vital rates from the latest revision of the UN's World Population Prospects which guaranteed our projections for 2010 were similar to the UN estimates for 2010 (UNPD 2011b).

Life expectancies for men and women are assumed separately, starting from the empirically given levels and are assumed to increase each by two years per decade until they reach the indicated level of maximum life expectancy - 90, 100, and 120 in the three scenarios. Because of different starting conditions, this maximum is reached at different points in time in different populations. After the maximum has been reached mortality is assumed to stay constant.

For fertility the indicated target level is assumed to be reached between 2030 and 2050 with linear interpolation between the current level and the target level. Here we distinguish between currently high and low fertility countries. For the low fertility regions (all parts of Europe, North America, Pacific OECD, and the China region) we assumed that the target level will be reached by 2030. For all other world regions we assumed that the indicated target level will be reached in 2050. For Africa, which currently has the highest level of fertility, two additional special scenarios have been calculated, one in which the target is only reached in 2070 and one in which fertility is assumed to remain stalled at a TFR of 5.0. For the last scenario the results are only presented to 2200 because they would quickly reach impossibly high levels.

For migration we made similar assumptions to the projections employed by the UN and those of the International Institute for Applied Systems Analysis (IIASA) 
which for convenience assume a slow phasing out of international migration. This is clearly unrealistic, particularly in view of the possible consequences of climate change, but has little effect on global population size, which is the main point of interest here. As such, we do not present the regional projections, rather a global aggregation.

Tables in the supplementary information list the results for selected years in terms of total population size and the proportion of the population above the age of 80 . The labels for each column first give the target TFR and then after a hyphen the maximum life expectancy at birth which is assumed for the listed scenario.

\section{Results}

Figure 1 illustrates the longer term sensitivity of population size to even very small differences in fertility levels according to three different scenarios of life expectancy. In the 'central' mortality scenario of an asymptotic mean life expectancy of 100 years, by 2100 the difference between TFRs of 2.0 and 1.75 already amounts to almost 2 billion, increasing to over 5 billion by 2200 and 7 billion by 2300. A TFR of 2.25, on the other hand, would lead to continued massive growth reaching around 20 billion in 2200 and 30 billion in 2300. A TFR of 2.5 would result in a global population of 77 billion by 2300.

If, however, global fertility in the long run converged to a level of 1.5 - which is slightly below the 2009 average level in the European Union of 1.59 (Eurostat 2012) then, after peaking around the middle of the century, the world population would return to the current level of seven billion people by 2100 . By the end of the $22^{\text {nd }}$ century it would then fall below three billion even though under this scenario, life expectancies would continue to increase until they reach 100 years in all parts of the world. Still lower fertility assumptions, based upon the kind of figures currently seen in East Asia, would result in more rapid declines and by 2200 in total world population sizes around one billion or below. The exact numbers for all scenarios, as well as the implications in terms of the age structures of the population, are listed in the appendix.

A final observation can be made concerning the relatively marginal contribution of changes in life expectancy to the overall quantum compared to the impact of alternate future patterns of fertility. Though this is demographically obvious, the relative insensitivity of the global sums to different patterns of mortality change is still worth highlighting. 
Figure 1: Global population size from 2000 to 2300 resulting from alternative global fertility levels as indicated (TFR to be reached by 2030-2050 and then kept constant) combined with a maximum life expectancy of (a) 90, (b) 100 and (c) 120 years

(a)

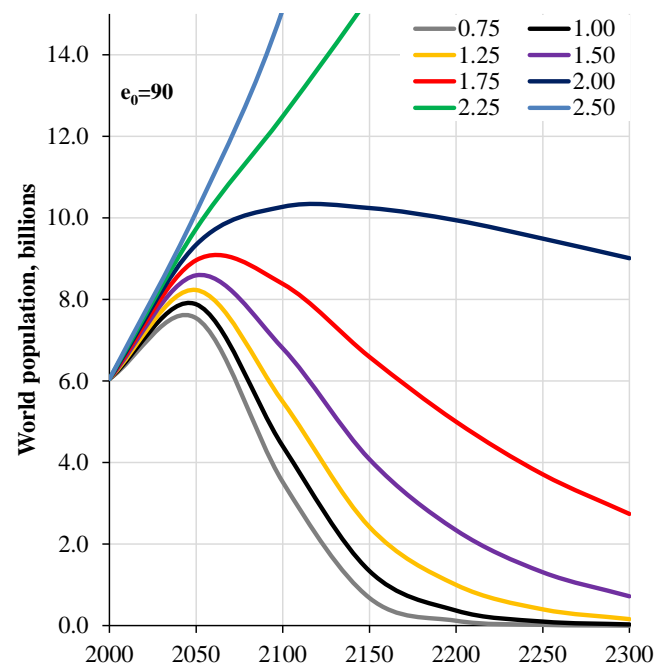

(b)

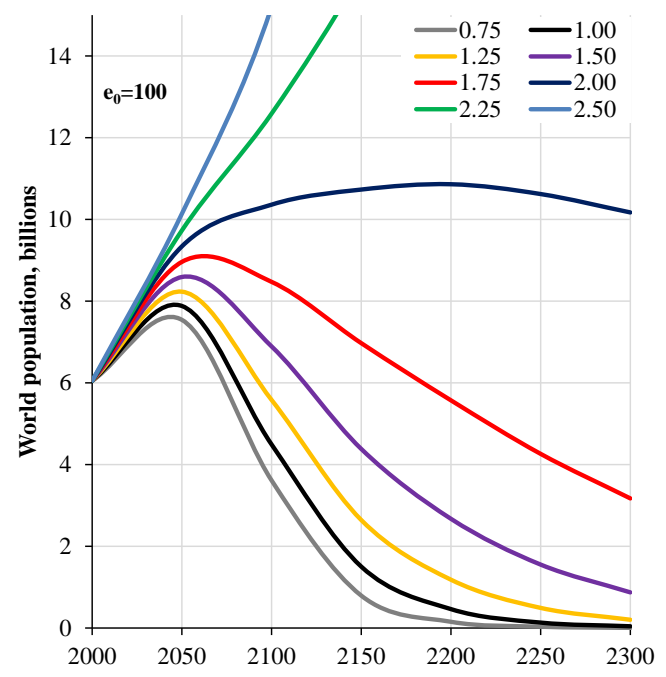




\section{Figure 1: (Continued)}

(c)

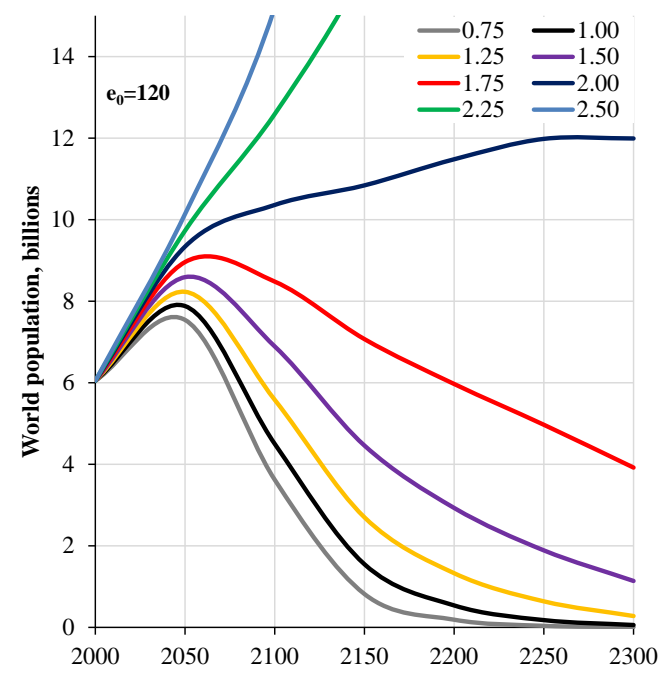

Note: For sub-Saharan Africa, special stalled fertility decline scenarios were defined. For mortality, further improvements in life expectancy at two years per decade were assumed up until a certain maximum level. Maximum life expectancy was assumed to be 100 years (for listing of full set of results from alternative scenarios see supporting online text).

\section{Conclusions}

We suggest the dominant two-child norm may not necessarily be the end point of transition as sizeable human populations exist where the voluntarily chosen ideal family size is heavily concentrated on just one child per woman with TFRs as low as 0.6-0.8. We have demonstrated the significant long term implications of possible sustained low fertility levels by producing the first global population projections into the twentysecond and twenty-third centuries based on a broader range of alternative fertility levels.

A global move to the fertility levels seen in a number of Chinese urban centres (around 0.75 ) over the coming 40 years would result in a peaking of global population before 2050 and a decline to only 3.6 billion in 2100 and 150 million people by 2200 . But even the more realistic range of long term fertility levels of 1.5-1.75 (higher than it has been in much of Europe for the past decades) would lead to declines in global population size of 2.6-5.6 billion by 2200 and even 0.9-3.2 billion by 2300. Therefore, 
even under conditions of further substantial increases in life expectancy, world population size would decline significantly if the world, in the longer run, followed the examples of Europe and East Asia.

The one continent where such a future looks most doubtful is Africa. In several sub-Saharan African countries the fertility decline is not proceeding well or may even have stalled (Ezeh, Mberu, and Emina 2009, Bongaarts 2008). For this reason we calculated a number of additional scenarios for Sub-Saharan Africa with a population of currently around 700 million (see supporting materials, Appendix 2). If the target fertility levels are only reached by 2070 instead of 2050, a TFR of 2.0 would result in 2.9 billion instead of 2.0 billion in 2100. If fertility remained completely stalled around the current fertility level of 5.0 already in 2100, the population size of Sub-Saharan Africa would reach a stunning 12 billion and a sheer impossible 355 billion by 2200 . If fertility continued to stay at such high levels it is not implausible to assume that population growth would be checked through increasing mortality. It is worth noting that future decline in Africa is far from being a certainty and that, in analogy to the above described 'Low Fertility Trap', there may be a high fertility trap mechanism in which rapid population growth inhibits the development that would bring down fertility (Dasgupta 1993; King 1990). Past experience has shown that the best way to break this vicious circle is the combination of female education with provision of family planning services (see, for example, Bongaarts and Sinding 2011; Lutz 2009; and Jejeebhoy 1996).

Is a future with long term global fertility below two and, hence, long term population decline something to be concerned about? Ecologists have long demanded a smaller world population size with a lighter ecological footprint and assumed, in the spirit of Malthus, that this will come naturally as a consequence of higher mortality caused by overpopulation and the resulting disasters. Our calculations clearly demonstrate that this desired decline can be reached even under conditions of further increasing life expectancy.

What about the problems of rapid population aging resulting from low fertility? In the long run, the age structure will stabilize under constant fertility and mortality rates even when the population is shrinking. The tables in the online supporting materials clearly illustrate this for the proportion above the age of 80 which eventually stabilizes. But over the coming decades the still accelerating speed of ageing (Lutz, Sanderson, and Scherbov 2008) seems to be the main problem because it requires a higher speed of adjusting social support and pension systems. Here the good news, however, lies in the on-going trend of improving health among the elderly, which can be assumed to continue into the future. Furthermore, if education and human capital is factored in, it has recently been suggested that a fertility level of 1.6-1.8 could be optimal even from an age-dependency perspective, if the smaller cohorts are better educated and therefore 
more productive (Lutz, Basten, and Striessnig 2012). Hence in the future the focus on population size and age structure should be complemented by one on education and health (Cohen 2008; Lutz, Goujon, and Doblhammer-Reiter 1998). Finally, it is likely that these fewer and more empowered people would be better able to cope with, and adapt to, climate change.

\section{Acknowledgements}

We thank participants who gave helpful comments at presentations in Vienna and Dallas. Funding for this work was made possible by the European Research Council (ERC) Advanced Investigator Grant focusing on "Forecasting Societies' Adaptive Capacities to Climate Change" (ERC-2008-AdG 230195-FutureSoc); and the KONE Foundation, "Epävarma perheellistyminen: nuoret ja väestödynamiikka kolmessa maanosassa”, Grant number 081433. 


\section{References}

Abrahamson, P., Boje, T.P., and Greve, B. (2005). Welfare and families in Europe. Aldershot: Ashgate.

Basten, S. (2013). Comparing projections assumptions of fertility in advanced Asian economies; or 'thinking beyond the medium variant'. Asian Population Studies, (forthcoming).

Basten, S., Coleman, D., and Gu, B. (2012). Re-examining the fertility assumptions in the UN's 2010 World Population Prospects: Intentions and fertility recovery in East Asia? Paper presented at Population Association of America Annual Meeting, San Francisco, CA, May 52012.

Basten, S. and Kumara, M. (2011). The development of one-child norms in Asia; or, thinking beyond 1.85. Paper presented at Workshop on childbearing and evolutionary theory, St. John's College, Oxford, UK, April 142011.

Boling, P. (2008). Demography, culture, and policy: Understanding Japan's low fertility. Population and Development Review 34(2): 307-326. doi:10.1111/ j.1728-4457.2008.00221.x.

Bongaarts, J. (2008). Fertility transitions in developing countries: Progress or stagnation? Studies in Family Planning 39(2): 105-110. doi:10.1111/ j.1728-4465.2008.00157.x.

Bongaarts, J. and Sinding, S. (2011). Population policy in transition in the developing world. Science 333(6042): 574-576. doi:10.1126/science.1207558.

Branigan, T. (2009). Shanghai encourages second child for eligible couples. The Guardian [London, UK], Saturday 25th July.

Cohen, J.E. (2008). Make secondary education universal. Nature 456: 572-573. doi:10.1038/456572a.

Dasgupta, P. (1993). An inquiry into well-being and destitution. Oxford: Clarendon Press.

Eurostat (2012). Statistics database. [electronic resource]. Brussels: Eurostat. http://epp.eurostat.ec.europa.eu/portal/page/portal/eurostat/home.

Ezeh, A.C., Mberu, B.U., and Emina, J.O. (2009). Stall in fertility decline in Eastern African countries: Regional analysis of patterns, determinants and implications. Philosophical Transactions of the Royal Society B: Biological Sciences 364(1532): 2991-3007. doi:10.1098/rstb.2009.0166. 
Frejka, T. (2008a). Overview chapter 2: Parity distribution and completed family size in Europe. Incipient decline of the two-child family model. Demographic Research 19(4): 47-72. doi:10.4054/DemRes.2008.19.4.

Frejka, T. (2008b). Overview chapter 3: Birth regulation in Europe: Completing the contraceptive revolution. Demographic Research 19(5): 73-84. doi:10.4054/ DemRes.2008.19.5.

Frejka, T. and Sobotka, T. (2008). Overview Chapter 1: Fertility in Europe: Diverse, delayed and below replacement. Demographic Research 19(3): 15-46. doi:10.4054/DemRes.2008.19.3.

Frejka, T., Sobotka, T., Hoem, J.M., and Toulemon, L., (2008). Summary and general conclusions: Childbearing trends and policies in Europe. Demographic Research 19(2): 5-14. doi:10.4054/DemRes.2008.19.2.

Goldstein, J.R., Sobotka, T., and Jasilioniene, A. (2009). The end of "lowest-low" fertility? Population and Development Review 35(4): 663-699. doi:10.1111/ j.1728-4457.2009.00304.x.

Gu, B. (2009). The arrival of low fertility in China. In: Jones, G., Straughan, P.T., Chan, A. (eds.). Ultra-Low Fertility in Pacific Asia: Trends, Causes and Policy Issues. Abingdon, UK: Routledge: 73-95.

Hermalin, A.I. and Liu, X. (1990). Gauging the validity of responses to questions on family size preferences in China. Population and Development Review 16(2): 337-354. doi:10.2307/1971594.

HKCSD (2012). Hong Kong Population Projections 2012-2041. Hong Kong: Hong Kong Census and Statistics Department.

Hou, Y. and Ma, X. (2008). Studies of the desired bearing of one child in urban Beijing (in Chinese). Social Science of Beijing 9: 27-31.

Hou, Y., Ma, X., and Huang, K. (2008). Research on the fertility desire and behavior of Beijing urban women from only-child families (in Chinese). Population and Development (China) 14: 47-54.

ICF International (2012). MEASURE DHS STATcompiler [electronic resource]. Fairfax, VA: ICF International. http://www.statcompiler.com.

Jejeebhoy, S.J. (1996). Women's education, autonomy, and reproductive behaviour: Experience from developing countries. New York: Oxford University Press. 
King, M. (1990). Health is a sustainable state. The Lancet 336(8716): 664-667. doi:10.1016/0140-6736(90)92156-C.

Kohler, H.-P, Billari, F.C., and Ortega, J.A. (2002). The emergence of lowest-low fertility in Europe during the 1990s. Population and Development Review 28(4): 641-680. doi:10.1111/j.1728-4457.2002.00641.x.

Lutz, W. (2009). Sola schola et sanitate: Human capital as the root cause and priority for international development? Philosophical Transactions of the Royal Society B: Biological Sciences 364(1532): 3031-3047. doi:10.1098/rstb.2009.0156.

Lutz, W., Basten, S., and Striessnig, E. (2012). Optimal fertility. In: Kaufman, E. and Wilcox, B. (eds.). Whither the Child? Causes and Consequences of Low Fertility. Boulder, CO: Paradigm Press: 205-233.

Lutz, W., Goujon, A., and Doblhammer-Reiter, G. (1998). Demographic dimensions in forecasting: Adding education to age and sex. Population and Development Review 24(Supplement: Frontiers of Population Forecasting): 42-58.

Lutz, W., Sanderson, W., and Scherbov, S. (2004). The end of world population growth. In: Lutz, W., Sanderson, W., and Scherbov, S. (eds.). The end of world population growth in the 21st century: New challenges for human capital formation and sustainable development. London: Earthscan: 17-62.

Lutz, W., Sanderson, W., and Scherbov, S. (2008). The coming acceleration of global population ageing. Nature 451: 716-719. doi:10.1038/nature06516.

Lutz, W., Skirbekk, V., and Testa, M.R. (2006). The Low Fertility Trap Hypothesis: Forces that may lead to further postponement and fewer births in Europe. Vienna Yearbook of Population Research 2006: 167-192. doi:10.1553/ populationyearbook2006s167.

Morgan, S.P. and Rackin, H. (2010). The correspondence between fertility intentions and behavior in the United States. Population and Development Review 36(1): 91-118. doi:10.1111/j.1728-4457.2010.00319.x.

Nakicenovic, N. and Swart, R. (eds.) (2000). Special Report on Emissions Scenarios : A Special Report of Working Group III of the Intergovernmental Panel on Climate Change. Cambridge: Cambridge University Press.

National Bureau of Statistics of China (2011). Communiqué of the National Bureau of Statistics of People's Republic of China on Major Figures of the 2010 Population Census (No. 2). [electronic resource]. Beijing: NBS. http://www.stats.gov.cn/ english/newsandcomingevents/t20110429_402722516.htm. 
Scherbov, S. and Grechucha, V. (1988). "DIAL" - A System for Modeling Multidimensional Demographic Processes. Laxenburg, Austria: International Institute for Applied Systems Analysis (IIASA Working Paper WP-88-36). http://www.iiasa.ac.at/publication/more_WP-88-036.php.

SMPFPC (2008). Briefing on SMPFPC Fertility Survey 2008 (in Chinese). Shanghai: Shanghai Municipal Population and Family Planning Commission.

SMPFPC (2009a). 2008 Houshold population, age, fertility and early education (in Chinese). [electronic resource]. Shanghai: Shanghai Municipal Population and Family Planning Commission. http://rkjsw.sh.gov.cn/dr/stat/ssh/20090611/ 0000000035000410011343698.html.

SMPFPC (2009b). The registered population of Shanghai, total fertility rates (in Chinese) . [electronic resource]. Shanghai: Shanghai Municipal Population and Family Planning Commission. http://rkjsw.sh.gov.cn/dr/stat/ssh/2003-1008/0010783.html?openpath=spfp/stat/ssh.

SMPFPC (2009c). Born in Shanghai in 2009: The population forecast and analysis of the results of the survey release (in Chinese). Shanghai: Shanghai Municipal Population and Family Planning Commission.

Sobotka, T. (2004). Is lowest-low fertility in Europe explained by the postponement of childbearing? Population and Development Review 30(2): 195-220. doi:10.1111/j.1728-4457.2004.010_1.x.

Sobotka, T., Skirbekk, V., and Philipov, D. (2011). Economic recession and fertility in the developed world. Population and Development Review 37(2): 267-306. doi:10.1111/j.1728-4457.2011.00411.x.

Straughan, P., Chan, A., and Jones, G. (2008). Ultra-low fertility in Pacific Asia: Trends, causes and policy issues. Abingdon, UK: Routledge.

Testa, M.R. (2012). Family sizes in Europe: Evidence from the 2011 Eurobarometer Survey. Vienna: Vienna Institute of Demography (VID European Demographic Research Papers; 2012:2). http://www.oeaw.ac.at/vid/download/ edrp_2_2012.pdf.

UNFPA (2011). Impact of demographic change in Thailand. Bangkok: United Nations Population Fund.

UNPD (2003). World Population Prospects: The 2002 revision highlights. New York: United Nations Population Division. (ESA/P/WP. 180) http://www.un.org/esa/ population/publications/wpp2002/WPP2002-HIGHLIGHTSrev1.PDF. 
UNPD (2004). World population to 2300. New York: United Nations Population Division. (ST/ESA/SER.A/236).

UNPD (2011a). Assumptions underlying the 2010 Revision. New York: United Nations Population Division. http://esa.un.org/wpp/Documentation/pdf/ WPP2010_ASSUMPTIONS_AND_VARIANTS.pdf.

UNPD (2011b). World Population Prospects: The 2010 Revision. New York: United Nations Population Division. http://esa.un.org/wpp.

Wattenberg, B. J. (2005). Fewer: How the new demography of depopulation will shape our future. New York: Ivan R. Dee Publishers.

Wen, Y., Yin, Q., Shuai, Y., and Wen, Y. (2005). Analysis of birth preference of young generation in Nanjing (in Chinese). Northwest Population Journal 2: 10-13.

Wen, Y. and Zong, Z.-H. (2006). Birth preference of people at birth age in Changzhou city and its causes (in Chinese). Journal of Nanjing College for Population Programme Management 22: 40-43.

Zheng, Z., Cai, Y., Feng, W., and Gu, B. (2009). Below-replacement fertility and childbearing intention in Jiangsu Province. China Asian Population Studies 5(3): 329-347. doi:10.1080/17441730903351701. 


\section{Appendix 1: Detailed results of alternative global population projections to 2300}

The calculations as presented in this paper have all been carried out at the level of 13 world regions. These regions as listed below follow the classification as used in the Intergovernmental Panel on Climate Change's 2000 SRES emissions scenarios 'Special Report on Emissions Scenarios' (Nakicenovic and Swart 2000). A detailed listing of which countries belong to which region is given in which also documents the starting data for the jump-off year 2000 (Lutz et al. 2004). The scenarios have been calculated on the basis of regular cohort-component projections by age (single years of age) and sex in single-year time steps up to 2300.

The following tables list the results for selected years in terms of total population size and the proportion of the population which is above the age of 80 . The labels for each column first give the target Total Fertility Rate (TFR) and then after a hyphen the maximum life expectancy at birth which is assumed for the listed scenario. Life expectancies for men and women are assumed separately, starting from the empirically given levels and are assumed to increase each by two years per decade until they reach the indicated level of maximum life expectancy. Because of different starting conditions, this maximum is reached at different points in time in different populations. After the maximum has been reached mortality is assumed to stay constant.

For fertility, the indicated target level is assumed to be reached between 2030 and 2050 with linear interpolation between the current level and the target level. Here we distinguish between currently high and low fertility countries. For the low fertility regions (all parts of Europe, North America, Pacific OECD and the China region) we assumed that the target level will be reached by 2030. For all other world regions we assumed that the indicated target level will be reached in 2050. For Africa, which currently has the highest level of fertility, two additional special scenarios have been calculated, one in which the target is only reached in 2070 and one in which fertility is assumed to remain stalled at a TFR of 5.0. For the last one the results are only listed to 2200 because they would quickly reach impossibly astronomic magnitudes.

For migration, we made similar assumptions to the UN and IIASA projections which for convenience assume a slow phasing out of international migration. This is clearly unrealistic, particularly in view of the possible consequences of climate change, but has little effect on global population size which is the main point of interest here.

Figures S1 and S2 below show the same pictures as the one given in the main text for a maximum life expectancy of 100 years for the scenarios that assume maximum life expectancies of 90 and 120 years, respectively. 


\section{Supplementary Tables}

In the following tables all the population sizes are listed in billions.

Table S1: Tables of resulting population size - Scenarios with maximum life expectancy of 90

\begin{tabular}{ccccccccc}
\hline World & & & & & & & & \\
& $\mathbf{0 . 7 5 - 9 0}$ & $\mathbf{1 . 0 0 - 9 0}$ & $\mathbf{1 . 2 5 - 9 0}$ & $\mathbf{1 . 5 0 - 9 0}$ & $\mathbf{1 . 7 5 - 9 0}$ & $\mathbf{2 . 0 0 - 9 0}$ & $\mathbf{2 . 2 5 - 9 0}$ & $\mathbf{2 . 5 0 - 9 0}$ \\
\hline 2000 & 6.05 & 6.05 & 6.05 & 6.05 & 6.05 & 6.05 & 6.05 & 6.05 \\
2050 & 7.54 & 7.88 & 8.23 & 8.59 & 8.96 & 9.34 & 9.73 & 10.14 \\
2100 & 3.52 & 4.40 & 5.49 & 6.80 & 8.38 & 10.27 & 12.49 & 15.11 \\
2150 & 0.68 & 1.34 & 2.42 & 4.08 & 6.59 & 10.24 & 15.43 & 22.65 \\
2200 & 0.12 & 0.37 & 1.00 & 2.34 & 5.00 & 9.94 & 18.67 & 33.45 \\
2250 & 0.02 & 0.10 & 0.40 & 1.31 & 3.71 & 9.49 & 22.28 & 48.83 \\
2300 & 0.00 & 0.03 & 0.16 & 0.72 & 2.74 & 9.01 & 26.47 & 71.04 \\
\hline
\end{tabular}

Table S2: Tables of resulting population size - Scenarios with maximum life expectancy of 100

\begin{tabular}{ccccccccc}
\hline World & & & & & & & & \\
& $\mathbf{0 . 7 5 - 1 0 0}$ & $\mathbf{1 . 0 0 - 1 0 0}$ & $\mathbf{1 . 2 5 - 1 0 0}$ & $\mathbf{1 . 5 0 - 1 0 0}$ & $\mathbf{1 . 7 5 - 1 0 0}$ & $\mathbf{2 . 0 0 - 1 0 0}$ & $\mathbf{2 . 2 5 - 1 0 0}$ & $\mathbf{2 . 5 0 - 1 0 0}$ \\
\hline 2000 & 6.05 & 6.05 & 6.05 & 6.05 & 6.05 & 6.05 & 6.05 & 6.05 \\
2050 & 7.54 & 7.88 & 8.23 & 8.59 & 8.96 & 9.34 & 9.73 & 10.14 \\
2100 & 3.61 & 4.49 & 5.58 & 6.89 & 8.47 & 10.36 & 12.59 & 15.20 \\
2150 & 0.79 & 1.50 & 2.64 & 4.38 & 6.97 & 10.73 & 16.03 & 23.39 \\
2200 & 0.15 & 0.46 & 1.18 & 2.67 & 5.57 & 10.86 & 20.10 & 35.59 \\
2250 & 0.03 & 0.13 & 0.49 & 1.55 & 4.26 & 10.62 & 24.47 & 52.85 \\
2300 & 0.00 & 0.04 & 0.20 & 0.87 & 3.17 & 10.17 & 29.30 & 77.46 \\
\hline
\end{tabular}


Table S3: Tables of resulting population size - Scenarios with maximum life expectancy of 120

\begin{tabular}{ccccccccc}
\hline World & & & & & & & & \\
& $\mathbf{0 . 7 5 - 1 2 0}$ & $\mathbf{1 . 0 0 - 1 2 0}$ & $\mathbf{1 . 2 5 - 1 2 0}$ & $\mathbf{1 . 5 0 - 1 2 0}$ & $\mathbf{1 . 7 5 - 1 2 0}$ & $\mathbf{2 . 0 0 - 1 2 0}$ & $\mathbf{2 . 2 5 - 1 2 0}$ & $\mathbf{2 . 5 0 - 1 2 0}$ \\
\hline 2000 & 6.05 & 6.05 & 6.05 & 6.05 & 6.05 & 6.05 & 6.05 & 6.05 \\
2050 & 7.54 & 7.88 & 8.23 & 8.59 & 8.96 & 9.34 & 9.73 & 10.14 \\
2100 & 3.62 & 4.50 & 5.58 & 6.89 & 8.48 & 10.36 & 12.59 & 15.20 \\
2150 & 0.82 & 1.55 & 2.70 & 4.46 & 7.07 & 10.84 & 16.16 & 23.53 \\
2200 & 0.19 & 0.54 & 1.33 & 2.93 & 5.97 & 11.48 & 20.99 & 36.83 \\
2250 & 0.04 & 0.18 & 0.64 & 1.89 & 4.97 & 11.98 & 26.91 & 56.99 \\
2300 & 0.01 & 0.06 & 0.28 & 1.14 & 3.92 & 11.99 & 33.34 & 85.77 \\
\hline
\end{tabular}

Table S4: IPCC world regions employed

\begin{tabular}{ll}
\hline North Africa & China \& CPA \\
Sub-Saharan Africa & Pacific Asia \\
North America & Pacific OECD \\
Latin America & Western Europe \\
Central Asia & Eastern Europe \\
Middle East & Former Soviet Union \\
South Asia & \\
\hline
\end{tabular}

Table S5: Resulting total population size in billions of special scenario for SubSaharan Africa in which the indicated target fertility level is reached in 2070 instead of 2050. Combined with a maximum life expectancy of 100 years. Otherwise same assumptions as above.

\begin{tabular}{rcccccccr}
\hline & $\mathbf{0 . 7 5 - 1 0 0}$ & $\mathbf{1 - 1 0 0}$ & $\mathbf{1 . 2 5 - 1 0 0}$ & $\mathbf{1 . 5 - 1 0 0}$ & $\mathbf{1 . 7 5 - 1}$ & $\mathbf{2 - 1 0 0}$ & $\mathbf{2 . 2 5 - 1 0 0}$ & $\mathbf{2 . 5 - 1}$ \\
\hline 2000 & 0.61 & 0.61 & 0.61 & 0.61 & 0.61 & 0.61 & 0.61 & 0.61 \\
2050 & 1.67 & 1.72 & 1.76 & 1.81 & 1.85 & 1.9 & 1.95 & 1.99 \\
2100 & 1.35 & 1.58 & 1.85 & 2.16 & 2.51 & 2.9 & 3.34 & 3.83 \\
2150 & 0.36 & 0.61 & 0.96 & 1.46 & 2.14 & 3.05 & 4.25 & 5.81 \\
2200 & 0.08 & 0.21 & 0.47 & 0.96 & 1.81 & 3.22 & 5.49 & 8.99 \\
2250 & 0.02 & 0.08 & 0.24 & 0.64 & 1.55 & 3.44 & 7.12 & 13.97 \\
2300 & 0 & 0.02 & 0.1 & 0.37 & 1.19 & 3.34 & 8.56 & 20.35 \\
\hline
\end{tabular}


Table S6: Resulting total population size (in billions) of special scenario for Sub-Saharan Africa in which the fertility level is kept constant at $\mathbf{5 . 0}$ from 2010 onwards. Combined with a maximum life expectancy of 100 years.

\begin{tabular}{cccccc}
\hline Year & $\mathbf{2 0 0 0}$ & $\mathbf{2 0 5 0}$ & $\mathbf{2 1 0 0}$ & $\mathbf{2 1 5 0}$ & $\mathbf{2 2 0 0}$ \\
\hline Population (bn) & 0.61 & 2.5 & 12.27 & 64.95 & 355.3 \\
\hline
\end{tabular}

\section{Appendix 2: Input data, code and method of calculation}

The input data for the projections are available from the authors or from the Demographic Research editorial office.

Despite both the commitment of the authors and the journal to sharing code, in this case it is somewhat problematic. The calculations in this paper were made with a proven multi-state projections program written two decades ago using EXE code, itself an elaboration of "DIAL" - a system for modeling multidimensional demographic processes (Scherbov and Grechucha 1988). The program was compiled from FORTRAN and is extremely fast. For many years it was very important because running a multi-regional probabilistic model was very time consuming. Running this program on an arbitrary computer will be extremely complex without several special setups. This program is the core. But to run this core, we also use programs written some time ago in $\mathrm{C}$ and we also now use EXE code. This all runs further in batch mode. All the results are written to a special database format, which we specially developed years ago before the era of Microsoft Access to suit our special purposes and structure of the data. This database is several times more compact then MS Access. Using special C programs also written about 15-17 years ago we unpack this database into an ASCII format and then use R programs to present the results. And finally, scenarios are generated in Visual Basic in Excel, since they have to come into the model in a special format. In addition, there should be also several DLL libraries (which were written about 15 years ago) installed on the computer to run the programs. These files may not be compatible with Windows above 2003. 
Basten, Lutz \& Scherbov: Very long range global population scenarios 